Kocovska, E., Wilson, P., Young, D., Wallace, A.M., Gorski, C., Follan, M., Smillie, M., Puckering, C., Barnes, J., Gillberg, C., and Minnis, H. (2013) Cortisol secretion in children with symptoms of reactive attachment disorder. Psychiatry Research, 209 (1). pp. 74-77. ISSN 0165-1781

Copyright (c) 2012 Elsevier.

A copy can be downloaded for personal non-commercial research or study, without prior permission or charge

The content must not be changed in any way or reproduced in any format or medium without the formal permission of the copyright holder(s)

When referring to this work, full bibliographic details must be given

http://eprints.gla.ac.uk/86279/

Deposited on: 3 March 2014

Enlighten - Research publications by members of the University of Glasgow http://eprints.gla.ac.uk 


\title{
Cortisol secretion in children with symptoms of Reactive
}

\section{Attachment Disorder}

\begin{abstract}
Maltreated children with Reactive Attachment Disorder (RAD) have severe problems with social relationships and affect regulation. An association between early maltreatment and changes in the daily rhythm of cortisol secretion has already been reported for maltreated toddlers. We sought to find out whether such changes were apparent in school-age children with symptoms of RAD, who had experienced early maltreatment but were currently adopted in well functioning families. We recruited 66 children: 34 5-12 year old adopted children with an early history of maltreatment and with social difficulties such as indiscriminate friendliness; and 32 age- and sex-matched comparison children with no history of maltreatment or social difficulties. Daily rhythms of cortisol production were determined from saliva samples collected over two days. The adopted group had significantly lower absolute levels of cortisol compared to the control group, but a typical profile of cortisol secretion. There was no association between cortisol secretion and symptom scores for psychopathology.

Keywords

Maltreatment; adoption, indiscriminate friendliness, Reactive Attachment Disorder
\end{abstract}




\section{Introduction}

Reactive Attachment Disorder (RAD) is a serious disorder of social functioning associated with abuse and neglect. The Disinhibited subtype is characterised by indiscriminate friendliness which can be persistent and it has been suggested that this may be associated with personality problems in adult life (Lyons-Ruth, 2008). We have previously shown that children with indiscriminate friendliness can have complex neurodevelopmental problems and yet are frequently not engaged with therapeutic services (Kocovska et al., 2012). Sufferers can have problems with emotional and behavioural functioning and affect regulation ( Zeanah et al., 2002; Green 2003; Rutter and Sonuga-Barke, 2010; Gleason et al., 2011), and research with international (mainly post-intitutionalised) adoptees has demonstrated an association between indiscriminate friendliness and abnormalities of the Hypothalamo-Pituitary-Adrenal (HPA) axis, which governs the secretion of stress hormones such as cortisol (Johnson et al., 2011).

RAD is one of only two disorders (the other being Post Traumatic Stress Disorder, PTSD) that are directly associated, in the psychiatric classification systems, with aetiology: it is recommended that a diagnosis of RAD should not be made unless there is a history of maltreatment in the first 5 years of life (World Health Organisation, 2007; American Psychiatric Association, 2000). However, not all children who have experienced maltreatment in early life develop RAD and both genetic and environmental factors contribute to causality (Minnis et al., 2007). Because of the recognised association, in institutionalised children, between indiscriminate friendliness and abnormalities of the HPA axis, we have decided to focus, in this study, on the subgroup of maltreated children who are indiscriminately friendly. 
Maltreatment in early life can be associated with long-term changes in regulation of the stress hormone cortisol and we have previously published a systematic review of this literature (Hunter et al., 2011). Our review has demonstrated that studies of adults who have been maltreated show conflicting results regarding the impact of maltreatment on diurnal secretion of cortisol and that there is a gap in the literature as regards cortisol production in maltreated schoolage children (Hunter et al., 2011). Altered function of the HPA axis has been described in depression (Holsboer, 2001), post-traumatic stress disorder (Yehuda, 2009), ADHD (Freitag et al., 2009; Stadler et al., 2011), conduct disorder and antisocial personality disorder (Vanyukov et al., 1993). Studies of children reared in institutions have shown that lack of caregiver sensitivity and positive regard is associated with a general suppression of the hypothalamic-pituitary axis (Johnson and Gunnar, 2011) and that this can recover if the environment improves. For example, in 2006 Dozier and colleagues (Dozier et al., 2006) reported alterations in the production of salivary cortisol in maltreated toddlers in foster care and substantial recovery has been demonstrated with environmental improvement (Dozier et al., 2006; Tarullo and Gunnar, 2006). There is, however, no available research indicating the extent to which these changes can persist into middle childhood and the issue has not been explored in non-institutionalised schoolage children.

During development, cortisol production is modulated in in response to the social environment of the child (Tarullo and Gunnar, 2006;Dozier et al., 2008; Hunter et al., 2011). By school-age, under optimal conditions, cortisol levels peak in the morning about 30 minutes after waking, followed by a gradual decrease towards evening (Gunnar and Donzella, 2002). In an environment of persistent deprivation, neglect or abuse, the development of an infant's daily 
cortisol rhythm and/or stress reactivity may differ from that of other children in various ways (Brand et al. 2010). When production is persistently elevated or suppressed, or inadequately regulated in response to stress, there may be long-term consequences including effects on brain functioning (Caldji et al., 2000; Hunter et al.,, 2011). These may be associated with difficulties in handling stressful situations and predispose individuals towards developing mental health problems later in life (Teicher et al., 2003).

In this study, we examined the daytime pattern of cortisol production in previously maltreated children aged 5-12 years with symptoms of Disinhibited RAD who were currently adopted in well functioning families and comparison children without such difficulties. Our hypothesis was that, in these children, maltreatment in early life may have resulted in differences in cortisol secretion which persisted into school-age.

\section{Methods}

The study protocol was approved by the West of Scotland 2 NHS ethics committee.

2.1 Statistical power An a priori power calculation based on Dozier 2006 (Dozier et al., 2006) indicated that a sample size of 10 in each group would have a $90 \%$ power to detect a difference in means of -0.2 assuming that the common standard deviation is 0.13 using a two group t-test with a 5\% two sided significance level. In order to ensure this computation was not too conservative and to allow for the suspected non-parametric nature of the data, we recruited a larger sample. 


\subsection{Participants}

Because we were interested in potentially persistent effects of early life maltreatment in children with RAD symptoms, we needed to recruit a group of children with RAD symptoms who had experienced abuse and neglect within the early years, but who had not continued to live in these adverse circumstances. In the UK, the mean age of adoption is 4 years (http://www.groscotland.gov.uk/press/news2004/03adopt-press.html;

http://www.baaf.org.uk/info/stats/england.shtml) and the overwhelming majority of adopted children have experienced severe abuse and/or neglect in the early years prior to coming into

care. In general, they then reside in well-functioning families (Rushton et al., 2006). We therefore considered adopted school-age children with RAD symptoms to be the ideal group within which to test our hypothesis.

Adopted children were recruited via the charity 'Adoption UK': eligibility criteria were discussed with the Scottish Director who contacted all eligible families known to the charity living within travelling distance of the University. Children were eligible for inclusion if they were aged 5-12 years, had the core symptom of the Disinhibited form of RAD - indiscriminately friendly behaviour - plus a history of maltreatment. We did not attempt to recruit children with Inhibited RAD because it is thought to be extremely rare (Gleason et al., 2011) and we were unlikely to have enough inhibited children to make a meaningful distinction between the two subtypes in this study. Disinhibited RAD symptoms were verified using the Relationships Problems Questionnaire (RPQ): all participating adopted children had at least two disinhibited RAD symptoms on the RPQ (see below). Potentially participating children were excluded if they had moderate or severe intellectual disability (which can itself be associated with disinhibition), 
current family instability or ongoing maltreatment. Forty-three children were referred and 40 met inclusion criteria but one female child was subsequently excluded as she had signs of having reached puberty. Two families (five children) withdrew. Thirty-four children (18 boys and 16 girls; mean (SD) age 9.4 (1.8) years were clinically assessed. All adopted children were white British except for one child who was African American by birth. There were 13 sibling pairs and 1 sibship of 3 involved in the study. Because, in previous research, we have found that there is a very small correlation in scores between maltreated siblings living in the same substitute family (Minnis et al., 2001) we have not made statistical adjustments for clustering.

Comparison children were included if they were aged 5 - 12 years and excluded if they had any known child psychiatric diagnosis, moderate or severe intellectual disability, any history (even suspected) of child maltreatment, known contact with social work, child protection registration or any trauma within the last year (assessed using a brief screening instrument for acute stress, the Life Change Scale, Foxman, 2004). We ensured that no comparison children had high scores suggestive of RAD (i.e. over 6 on Relationship Problems Questionnaire - see later). Children were selected through two general medical practices in Glasgow. The practices had 750 children within the age range 5-12 years and 615 were eligible according to the inclusion criteria. The general practitioners sent 461 invitation letters: 58 responded, 9 withdrew and, because of gender and age mismatches, not all remaining eligible children were invited to participate. In order to address imbalances in age and gender, a further 62 invitation letters were re-sent to nonresponders from the original 461, this time only to families with boys aged 6-10 years. Of these, four had moved away, six responded and were assessed. The comparison group eventually 
comprised 32 children (17 boys and 15 girls; mean (SD) age 8.7 (2.4) years who were clinically assessed. All comparison children were white British.

Children in both the adopted and comparison group were offered a neuropsychiatric assessment using standardised tools for RAD, other diagnoses and measures of cognition and language functioning. These assessments have been described elsewhere (Kocovska et al., 2012).

\subsection{Procedure}

For adopted children, after obtaining consent forms from parents and children, an initial home visit was arranged during which demographic data were collected and parents were interviewed regarding the child's mental health and social functioning. For the comparison group, interviews were carried out either in the child's general practice or at home. Test tubes were delivered and instructions given regarding how to collect saliva samples three times a day over two days. A written instruction leaflet was left for each child (Patel et al., 2004).

\subsection{Measures}

Emotional and behavioural symptoms were measured using the Strengths and Difficulties Questionnaire (SDQ), a 25 item scale validated in large population research that covers conduct problems, emotional problems (depression/anxiety), hyperactivity, problems with peer relations and prosocial (caring, helpful) behaviour. The first four subscales are totalled to produce a Total Difficulties Score (Goodman et al., 2003). 
The Relationship Problems Questionnaire (RPQ) is a 10-item parent and teacher-report screening instrument for RAD symptoms. In a large general population twin sample, the RPQ had good internal consistency (Cronbach's alpha .85) and factor analysis identified that 6 items describe Inhibited RAD behaviours and 4 items describe Disinhibited RAD behaviours (Minnis et al., 2007).

Information about the adopted children's histories of abuse and/or neglect were gleaned from the "Form F" - the form completed by the social worker at the time of child placement in the adoptive family, using a standardised checklist developed for this study. The Form $\mathrm{F}$ is an unstructured form that contains as much detail as the social worker can glean about the child's pre-adoption history. From the Form F, we were able to extract information on the various types of abuse and neglect (emotional, physical and sexual), witnessing of domestic violence and parental history of drug and alcohol misuse.

\subsection{Saliva Sampling}

In a normal population the pattern of cortisol levels shows a peak in the morning, followed by sthe night value)(22). Saliva samples were therefore collected three times a day: morning: between $6-8$ am, 30 min after waking, before breakfast and before brushing teeth; mid-day: between $12-2 \mathrm{pm}$ before meal; evening: between $6-9 \mathrm{pm}$, just before going to bed and before brushing teeth. Children were asked to rinse their mouth with clear water 10 minutes before collection and then not to eat, chew or drink anything else prior to spitting directly into the collection tube (minimal volume of $1 \mathrm{ml}$ requested). Samples were stored in a freezer for up to 48 hours and sent to our department by post. The samples were then stored in the freezer at $-20^{\circ} \mathrm{C}$ 
and batches of around 120 delivered to the biochemistry laboratory for an analysis. Mucins were precipitated from saliva by a freeze thaw cycle followed by centrifugation. Cortisol was measured in an aliquot of the clear supernatant by a radioimmunoassay using ${ }^{125}$ I-cortisol as tracer (Patel et al., 2004).

Cortisol measures were averaged over the two daily readings for each of the subjects. Values were highly skewed and a logarithmic transformation came closest to normalising the data, resulting in a fairly symmetric distribution with descriptive means and medians being almost identical. A repeated measures analysis of variance was performed on the log transformed data and the model included a time by group interaction.

\section{Results}

\subsection{Sample description}

As can be seen in Table 1, the adopted children were a typically "late placed" sample, had all experienced maltreatment and had significantly higher symptom scores for both RAD and other forms of psychopathology.

Insert Table 1 about here

\subsection{Cortisol Secretion}


A repeated measures analysis of variances was performed on the log transformed data. There was strong evidence of a difference between the time points $(\mathrm{p}<0.001)$ and also between the groups ( $\mathrm{p}=0.047)$ although all cortisol results were within physiologically normal limits (see Figure 1). The time by group interaction was not significant $(\mathrm{p}=0.868)$ demonstrating that, despite overall differences in secretion, there was a similar diurnal pattern of secretion over time in adopted children compared to controls.

\section{Insert Figure 1 about here}

\subsection{Association between cortisol secretion and behaviour}

As expected, both SDQ and RPQ scores were significantly higher in the adopted group compared to the comparison group (see Table 1). There was no correlation between cortisol secretion in the morning, afternoon or evening and symptom scores for general psychopathology (SDQ) or Reactive Attachment Disorder (RPQ): Spearman's Rank Correlations ranged from $-.008(\mathrm{p}=0.96)$ to $-0.193 ;(\mathrm{p}=0.17)$.

\section{Discussion}

\subsection{Overview}

Primary-school aged children with RAD symptoms and a history of early maltreatment have a similar cortisol profile compared to controls, but with slightly lower levels of secretion. We did not detect any association between these small differences in cortisol secretion and social or behavioural difficulties. These small differences in secretion suggest the possibility that other 
aspects of the stress response system may be implicated in these children's social/emotional difficulties and this warrants further investigation. Various genetic and environmental factors affect the stress response system during development (Gunnar, 2007; Hunter et al., 2011) and it may be that, by middle childhood, cortisol secretion has become better regulated in the group that experienced early childhood maltreatment as a result of nurturing care by adoptive parents. It is also possible that there is a sensitive period for cortisol action in altering behaviour, and this period ends before primary school age, so HPA function normalises but with persistent effects on mental health.

\subsection{Limitations and directions for future research}

Our sample size was modest $(\mathrm{n}=66)$ although adequate according to a priori power calculations (Dozier et al., 2006). Only a modest proportion of our target population of typically developing children took part in the study, but we do not regard this as a major limitation as we set out to recruit a group of typically developing children, age and gender matched with our maltreated sample, and did not intend to recruit a sample representative of the general population. We did not use a standardised measure of pubertal status and this may useful in future studies. It would have been interesting to compare our sample of children with RAD symptoms with a sample of similarly maltreated children who did not have such symptoms, but as we did not have an estimate of the magnitude of differences in cortisol secretion in school-age children it would have been premature to design such a study. This would be an important next step and would demonstrate whether the reduced cortisol secretion is associated with symptoms of RAD, or is simply a broader index of maltreatment. We do not know whether and how the stress response system was altered during the severe maltreatment period in the early infancy/childhoods of 
these adopted children as their cortisol levels were not measured at that time, we did not have the opportunity to follow these children from infancy and only had retrospective data about children's maltreatment histories. In addition, cortisol responses can be either blunted or elevated in association with different environmental insults and it would be useful to investigate this in future larger studies. Prospective cohort studies of maltreated children, followed from early infancy, will be required to elucidate the probably complex relationships between specific types

of abuse and neglect, psychopathology and cortisol secretion through development. We were only able to test the basal activity of the HPA axis and not HPA reactivity to stressors: future research should encompass studies of cortisol reactivity to stress.

\subsection{Conclusion}

Cortisol secretion, in this sample of maltreated adopted children, was significantly lower than in comparison children but had a similar profile and was not associated with psychopathology.

\section{Acknowledgements}

The study is dedicated to the memory of Dr Mike Wallace, a highly valued collaborator who sadly died suddenly prior to the completion of data collection. Thanks are due to all participating families for their time and enthusiasm. We also wish to thank Ms Fiona Lettice, for helping us liaise with the adoptive families and for her comments on the conduct of the study, Ms Halina MacIntyre for conducting the laboratory analyses, Rachel Pritchett, Harriet Hockaday, Emma Lidstone, Diane Fraser and Charlotte Cuddihy for their help with data collection and Ms Irene O'Neill for administrative support. Funding: The study was supported financially by NHS Greater Glasgow and Clyde [Grant reference PN08 AD291]. 


\section{References}

American Psychiatric Association, 2000. Diagnostic and Statistical Manual of Mental Disorders (Fourth Edition Revised). American Psychiatric Association, Washington DC.

Brand, S., Brennan, P., Newport, D., Smith, A.K., Zachary, N., 2010. The impact of maternal childhood abuse on maternal and infant HPA axis function in the postpartum period.

Psychoneuroendocrinology 35 (5), 686-693.

Caldji, C., Diorio, J., and Meaney, M., 2000. Variations in maternal care in infancy regulate the development of stress reactivity. Biological Psychiatry 48 (12), 1164-1174.

Dozier, M., Manni, M., Gordon, M., Peloso, E., Gunnar, M., Stovall-McClough, K., Eldreth, D., Levine, S., 2006. Foster children's diurnal production of cortisol: An exploratory study. Child Maltreatment 11 (2), 189-197.

Dozier, M., Peloso, E., Lewis, E., Laurenceau, J., and Levine, S., 2008. Effects of an attachmentbased intervention on the cortisol production of infants and toddlers in foster care. Development and Psychopathology 20, 845-859.

Fisher, P., Gunnar, M., Dozier, M., Bruce, J., and Pears, K., 2006. Effects of therapeutic interventions for foster children on interventions for foster children on behavioral problems, caregiver attachment, and stress regulatory neural systems. Annals of the New Yorkhill Academy of Science 1094, 215-225.

Foxman P., 2004. The Worried Child: Recognizing Anxiety in Children and Helping Them Heal. Hunter House Publishers, Alameda, CA.

Freitag, C., Hanig, S., Palmason, H., Meyer, J., Wust, S., Seitz, C., 2009. Cortisol awakening response in healthy children and children with ADHD: Impact of comorbid disorders and psychosocial risk factors. Psychoneuroendocrinology 34, 1019-1028.

Gleason, M., Fox, N., Drury, S., Smyke, A., Egger, H., Nelson, C., Gregas, M., Zeanah, C., 2011. Validity of evidence-derived criteria for Reactive Attachment Disorder: indiscriminately social/disinhibited and emotionally withdrawn/inhibited types. Journal of the American Academy of Child and Adolescent Psychiatry 50 (3), 216-231.

Goodman, R., Ford, T., Simmons, H., Gatward, R., and Meltzer, H., 2003. Using the Strengths and Difficulties Questionnaire (SDQ) to screen for child psychiatric disorders in a community sample. International Review of Psychiatry 15 (1-2), 166-172.

Green, J., 2003. Are attachment disorders best seen as social impairment syndromes? Attachment and Human Development 5 (3), 259-264.

Gunnar, M., 2007. Stress Effects on the Developing Brain. In: Romer, D., Walker, E., (Eds.), Adolescent Psychopathology and the Developing Brain: Integrating Brain and Prevention Science. Oxford University Press, Oxford, pp. 127-147. 
Gunnar, M. and Donzella, B., 2002. Social regulation of the cortisol levels in early human development. Psychoneuroendocrinology 27, 199-220.

Holsboer, F., 2001. Stress, hypercortisolism and corticosteroid receptors in depress: implications for therapy. Journal of Affective Disorders 62 (1-2), 77-91.

Hunter, L., Minnis, H., and Wilson, P., 2011. Altered stress responses in children exposed to early adversity: A systematic review of salivary cortisol studies. Stress 14, 614-626.

Johnson, A., Bruce, J., Tarullo, A.,, Gunnar, M., 2011. Growth delay as an index of allostatic load in young children: Predictions to disinhibited social approach and diurnal cortisol activity. Development and Psychopathology 23, 859-871.

Johnson, D. and Gunnar, M., 2011. Growth failure in institutionalized children. Monographs of the Society for Research in Child Development 76 (4), 92-126.

Kocovska, E., Puckering, C., Follan, M., Smillie, M., Gorski, C., Barnes, J., Wilson, P., Young, D., Lidstone, E., Pritchett, R., Hockaday, H., Minnis, H., 2012. Neurodevelopmental problems in maltreated children referred with indiscriminate friendliness. Research in Developmental Disabilities 33, 1560-1565.

Lyons-Ruth, K., 2008. Contributions of the mother-infant relationship to dissociative, borderlines, and conduct symptoms in young adulthood. Infant Mental Health Journal 29 (3), 203-218.

Minnis, H., Pelosi A., Knapp, M. and Dunn, J., 2001. Mental health and foster carer training. Archives of Disease in Childhood, 84:302-306.

Minnis, H., Reekie, J., Young, D., O'Connor, T., Ronald, A., Gray, A., and Plomin, R., 2007. Genetic, environmental and gender influences on attachment disorder behaviours. British Journal of Psychiatry 190, 490495.

Patel, R., Shaw, S.R., McIntyre, H., McGarry, G., and Wallace, A., 2004. Morning salivary cortisol versus Synacthen test as a test of adrenal suppression. Annals of Clinical Biochemistry 41, 408-410.

Rushton, A., Monck, E., Upright, H., and Davidson, M., 2006. Enhancing adoptive parenting: devising promising interventions. Child and Adolescent Mental Health 11 (1), 25-31.

Rutter, M., Sonuga-Barke, E., Beckett, C., Castle, J., Kreppner, J., Kumsta, R., Schlotz, W., Stevens, S., Bell, C.A., Gunnar, M.., 2010. Deprivation-specific psychological patterns: Effects of institutional deprivation. Monographs of the Society for Research in Child Development 75, (1), 252.

Stadler, C., Kroeger, A., Weyers, P. Grasmann, D., Horschinek, M., Freitag, C., Clement, H., 2011 Cortisol reactivity in boys with attention-deficit/hyperactivity disorder and disruptive behavior problems: The impact of callous unemotional traits, Psychiatry Research 187 (2011), 204-209 
Tarullo, A. and Gunnar, M., 2006. Child maltreatment and the developing HPA axis. Hormones and Behavior 50, 632-639.

Teicher, M., Andersen, S., Polcari, A., Anderson, C., Navalta, C., and Kim, D., 2003. The neurobiological consequences of early stress and childhood maltreatment. Neuroscience and Biobehavioral Reviews 27, 33-44.

Vanyukov, M., Moss, H., Plail, J., Blackson, T., Mezzich, A., and Tarter, R., 1993. Antisocial symptoms in preadolescent boys and in their parents: Associations With Cortisol. Psychiatric Research 46, 9-17.

World Health Organisation, 2007. The ICD-10 International Statistical Classificastion of Diseases and Related Health Problems: Mental and Behavioural Problems, 10th Revision. World Health Organisation, Geneva.

Yehuda, R., 2009. Status of glucocorticoid alterations in post-traumatic stress disorder. Annals of the New York Academy of Sciences 1179, 56-69.

Zeanah, C., Smyke, A., and Dumitrescu, A., 2002. Attachment disturbances in young children. II: Indiscriminate behavior and instiutional care. Journal of the American Academy of Child and Adolescent Psychiatry 41, (8) 983-989. 
Table 1 - comparison of demographics, maltreatment history and symptom scores between adopted children and comparison children

\begin{tabular}{|c|c|c|}
\hline Variable & $\begin{array}{l}\text { Adopted } \\
\text { children (n } \\
=34)\end{array}$ & $\begin{array}{l}\text { Comparison } \\
\text { children }(n= \\
32)\end{array}$ \\
\hline \multirow[t]{2}{*}{ Gender (\%males) } & $51.5 \%$ & $43.1 \%$ \\
\hline & Mean (SD) & Mean (SD) \\
\hline Age (years) & $9.4(1.8)$ & $8.7(2.4)$ \\
\hline Age of adoption (in months) & $62.9(25.3)$ & $\mathrm{N} / \mathrm{A}$ \\
\hline Months with adoptive family & $51.3(26.8)$ & N/A \\
\hline Birth parent alcohol misuse & $74 \%$ & N/A \\
\hline Birth parent drug misuse & $62 \%$ & N/A \\
\hline $\begin{array}{l}\text { Physical and/or emotional neglect by } \\
\text { birth parent }\end{array}$ & $100 \%$ & N/A \\
\hline $\begin{array}{l}\text { History of physical abuse in birth } \\
\text { family }\end{array}$ & $49 \%$ & N/A \\
\hline $\begin{array}{l}\text { History of sexual abuse in birth } \\
\text { family }\end{array}$ & $20 \%$ & N/A \\
\hline
\end{tabular}

\begin{tabular}{lllcr}
\hline & & & $\begin{array}{c}\text { Statistical } \\
\text { Test }\end{array}$ & \\
\cline { 1 - 3 } $\begin{array}{l}\text { Total Difficulties Score on parent } \\
\text { SDQ }\end{array}$ & $20.1(7.8)$ & $7.59(6.03)$ & 6.83 & \\
Parent total RPQ score & $9.2(7.1)$ & $0.7(1.8)$ & 5.84 & $<.0001$
\end{tabular}




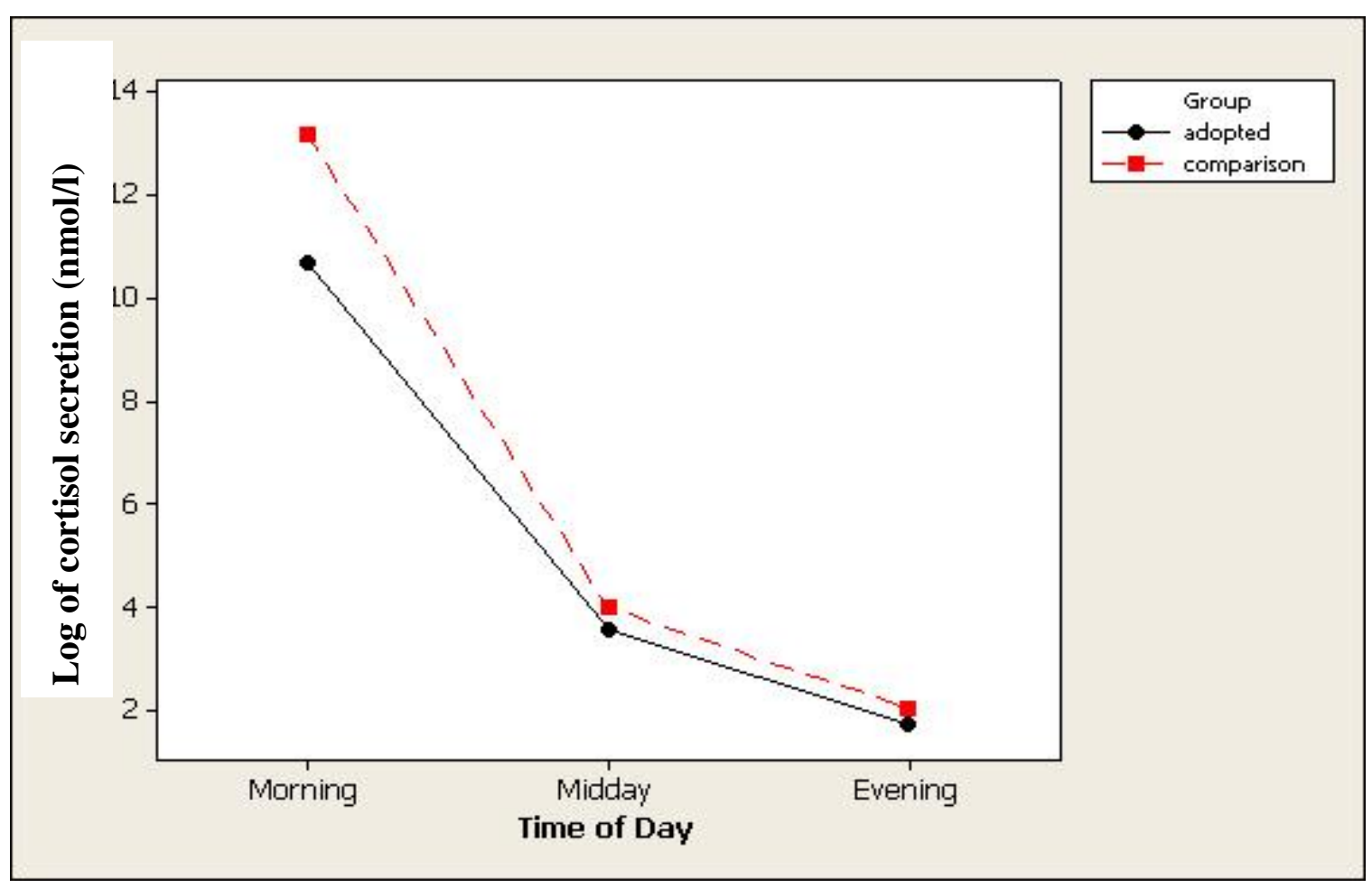

Figure 1- diurnal secretion of cortisol in adopted children and comparisons. There was a statistically significant difference between the groups $(p=0.047)$ but no time by group interaction ( $p=0.868)$ demonstrating that the profile of the groups was similar. 